\section{A review of the structure and function of vital registra- tion system in Ghana: towards improvement in mortality data quality for health policy analysis}

\author{
Julius N. Fobil,, 1,2,3 Eunice Aryee, ${ }^{1}$ \\ Francis Bilson, ${ }^{4}$ Juergen May, ${ }^{2}$ \\ Alexander Kraemer ${ }^{3}$
}

'School of Public Health, College of Health Sciences, University of Ghana; 'Bernhard-Notch Institute for Tropical Medicine, Hamburg, Germany; ${ }^{3}$ School of Public Health, Department of Public Health Medicine, Bielefeld University, Germany ${ }^{437}$ Military Teaching Hospital, Health Division, Ministry of Defense, Accra, Ghana

\section{Abstract}

Vital registration systems (VRS) are important in the collection of routine data on indicators of development. These are particularly useful if they are properly built to address weaknesses in the system leading to poor data quality. For instance, routine data on health events (e.g. morbidity, mortality etc.) are crucial for rapid assessment of disease burden and mortality trends in the population. They are also useful in the identification of vulnerable groups in populations. Despite their usefulness, VRS in many developing countries including Ghana are poorly structured raising questions about the quality of the output data from these systems. The present study aimed at assessing and documenting the structure and function of the VRS in Ghana, as well as at identifying the structural features that potentially compromise the reliability and validity of the output data from the Ghanaian VRS. To perform this study, collection and review of policy and legal documents establishing the VRS, documentation and evaluation of component structures of the system, assessment of procedural protocols guiding data collection processes and in-depth interviews with staff at the Ghana Births and Deaths Registry were performed. The assessment of the structure of the Ghana VRS, policy documents setting it up and the operational procedures reveals important lapses (e.g. presence of outmoded practices, imperfections in Births and Deaths Registry Act, 1965, Act 301 and imperfect system design) in the system that could compromise validity and reliability of the data generated from the VRS in Ghana.

\section{Introduction}

Information on health events is a crucial requirement for effective health policy planning. This is arguably why local and national health ministries in all countries invest heavy financial resources in demographic and health data collection. ${ }^{1,2}$ Most of the health data required for effective health policy planning in many low income countries are obtained from national Demographic and Health Surveys. However, some out of the ordinary health policy relevant information may also be obtained through various specialized epidemiological surveys such as crosssectional surveys and prospective studies.-8 Indeed, many of these surveys are very expensive requiring colossal amounts of money far greater than what national governments of least developed countries can afford, and thus calling for external donor support for their execution. Although several inexpensive routine data collection activities that generate vital information of relevance to health policy (e.g. information on live births, deaths, stillbirths, marriages, divorces, adoptions and important diseases) exist, these are in the state of near collapse or with weak structures. $^{2,9-12}$ For this reason, there are great concerns about the quality of the data generated from these feeble systems. But despite these concerns, it is widely acknowledged that if the structures of the systems are strengthened, they may yield information of sufficiently high quality for normal analytical work desired for national health policy planning and contextspecific reforms. ${ }^{8,13-18}$

In Ghana, the registration of vital events comes under the rubrics of the Births and Deaths Registry with a central office in Accra, the capital city, although there are other administrative data, which are collected by ministries and departments. The vital registration systems (VRS) in Ghana are characterized by inadequate registration facilities, weak structures and poor staffing. Despite the several benefits that can be achieved through a well established and well functioning vital registration system, the regrettable reality in Ghana is that the system is inefficient and does not cover up to half of the total events.

Although the vital registration system has operated in Ghana for more than a century, it is still based on outmoded practices and structures, which raise concerns about data quality, timeliness, and the lack of real-time linkage capabilities. This is largely because, the returns from the system are still stored and archived on paper cards and forms. This has been a matter of serious concern to the Registry, the nation and other international bodies, which rely on vital registration data for policy formulation and development planning.
Correspondence: Dr. Julius N. Fobil, School of Public Health, College of Health Sciences University of Ghana, P. 0. Box LG13, Legon.

E-mail: fobil@bnitm.de

Key words: vital registration system, routine data, reliability, validity, death cause, data quality.

Contributions: conception and design by JNF, JM \& AK. Data and material gathering were done by JNF, EA \& FB. Synthesis, drafting and editing of the manuscript were done all by authors.

Acknowledgements: funding for this study was generously provided by the Government of Ghana, through the GetFund.

Conflict of interest: the authors report no conflicts of interest.

Received for publication: 3 September 2010. Accepted for publication: 15 December 2010.

This work is licensed under a Creative Commons Attribution 3.0 License (by-nc 3.0)

(C) Copyright J.N. Fobil et al., 2011

Licensee PAGEPress, Italy

Journal of Public Health in Africa 2011; 2:e5 doi:10.4081/jphia.2011.e5

Registration of vital events in most rural areas in Ghana continues to drop sharply because of lack of registration facilities in those areas. For instance, in 2000, the Registry captured only $31 \%$ of total births expected for the year. In 2001 the coverage level dropped to $27 \%$ of expected births, while figures released for 2002 indicated an all time low level of $17 \%$. This is even worse for death, which reporting remains a low priority for most people because they have no motivation to report the death of relatives. Although there has been moderate and gradual improvement in the coverage of death reporting from 2000 to 2005 , the overall national annual figures are stunningly low (e.g. $20 \%$ in $2000,22 \%$ in $2001,21 \%$ in 2002 , $23 \%$ in 2003 and $24 \%$ in 2004).

In order to complement the weak VRS, provide more reliable data and to address health data needs for the study of population health in Ghana, the concept of demographic surveillance system (DSS) was introduced in Ghana. Starting first in Navrongo in 1993, subsequently in Kintampo and Dodowa, the DSS aimed to routinely update vital events (births, deaths, disease onset, migration, marriages, pregnancies, etc.) in the surveillance sites. A comparison of the frameworks on which both DSS and VRS operate and the objectives of both systems show striking similarities in component elements, i.e. both systems consist of structures and standard operating procedures to monitor vital events (births, deaths, migration, etc.) in 
well defined populations. ${ }^{19,20}$ Unlike censuses, describing the state of the population at a fixed point in time, vital statistics (in both VRS and DSS) are collected on a continuous basis, and thus are important for studying changes in population and health. ${ }^{21}$

Nonetheless, major structural differences exist between the Ghana VRS and DSS. For instance, whereas the DSS is constructed on robust epidemiological designs and attracting professionals from diverse backgrounds (i.e. highly qualified demographers, epidemiologists, sociologists, clinicians and economists) the VRS is more or less built on improperly designed political-administrative structures and is run by low level personnel. ${ }^{19,20}$ Perhaps these structural differences provide the basis for the difference in the quality of output data from the two systems. Although the DSS is regarded as a gold standard system in terms of the quality of output data which are preferred over those from the VRS, major challenges to the use of DSS data are that they do not come from a random sample of the reference population and by dint of their small population sizes (roughly 50 to 100,000 people) project overcrowding may overload host communities, blur project outcome measures and lead to wrong interpretations and false conclusions. ${ }^{19}$ This makes the operations of the DSS relatively easier to manage compared to the operations of the VRS which cover large populations i.e. populations of whole regions or whole districts. Implicitly, the establishment of satellite field centers in the districts could spread research load more evenly and disperse project overcrowding as held by Chandramohan and coworkers. ${ }^{20}$ The authors argue that if the proposed satellite field centers become coterminous with the existing political administrative set-up might lead to structural convergence of DSS and VRS, thus resolving the problem of VRS dysfunction in Ghana.

\section{Materials and Methods}

The overall goal of this synthesis was to assess and document the various procedures followed during mortality reporting in a view to identifying weaknesses and suggest ways (based on the DSS model) that could be applied to the VRS in Ghana to improve its output data.

Specific objectives were to: i) critically review relevant policy documents and technical reports regarding death reporting, medical cause of death certification and burials in Ghana; ii) assess and document the institutional structures and the administrative arrangements at the Death Registry in a view to discovering potential sources of weaknesses with potential adverse implications on the validity and reliability of output data; iii) make recommendations and suggestions as to how the VRS structures may be strengthened so that the inherent systematic biases be minimized.

The key assumption of this study design was that interviews with the Registrar and his assistants at the Central Registry in Accra would yield adequate and complete information on the overall structure and setup of Births and Deaths Registry. The scoped of the information derived, spanned from the conduct of the activities of the registration system to the potential weaknesses in the system that ultimately had implications on the overall quality of the data generated. This study employed a mixed strategy involving personal and indepth interviews with selected key personnel (the Registrar, some workers within the registry and sextons at the cemeteries).

As a first step, a comprehensive literature search, review and analysis of a compilation of policy documents, technical and non-technical reports, newsletters, research documents, and conference papers archived at the Ghana Ministry of Health, Births and Deaths Registry, mortuaries, hospitals, etc. that related to mortality reporting in Ghana were done. We conducted in-depth interviews as follows: i) staff of Births and Deaths Registry; ii) sextons overseeing the controlled cemeteries.

The in-depth interviews were held at personal level with the Registrar and 2 other staff members in the Central Office, 1 District Officer and 3 recorders of vital events in three different communities. The aim of the personal in-depth interviews was to provide grounds for comparison between what the prescribed duty was and what was actually done on the ground. Additionally, five sextons in 5 different controlled cemeteries were interviewed independently for the same reason.

\section{Findings from interviews and review of policy documents}

The section was written based upon a synthesis of the Registration of Births and Deaths Act, 1965 as amended by N.L.C.D. 285, 1968, Act 301, which established the Births and Deaths Registry, a host of related documents and interviews with the Registrar of Births and Deaths as well as other staff of the Registry. The section is divided into three themes, namely: the "structure of births and deaths registry", the "legal provisions" and the "death registration process" in Ghana. Under each thematic area, we documented what the provisions were and a discussion on implications on data quality.

\section{Structure of the Ghana births and deaths registry \\ Interviews with the Registrar and personnel}

at the Ghana births and deaths registry revealed that the activities of the vital registration system are coordinated from the Central Registry Office, located in Accra. Under a decentralized system, the entire country has been zoned into strata known as Registration Regions, which are coterminous with the political and administrative regions of the country. The Registration regions were reported to have been further subdivided into Registration Districts, which were also coterminous with the country's political administrative local authority areas. There is at least one Registry Office in each registration district, directed by the District Registration Officer, who supervises the registration system at sub-district level through to the registries and the reporting centers in the district (Figure 1).

Apart from the registries, our interviews revealed that there are also individual volunteers who record death events and report to the registries. Figure 1 shows the components and sub-components making up the Births and Deaths Registry in Ghana which are ordered hierarchically beginning with the basic units through to the Central Office. Under the current set-up, death registration is done in the communities by the staff of registries and in individual homes by registration assistants and volunteers respectively. In order to guard against double registration, source documents (medical certificate of cause of death or coroner's report) are collected from the persons presenting them and in return, burial permits are issued. A register is kept in the offices of the death registries. Death registration forms are then summarized into statistical summaries (aggregate totals by sex, age, etc) for purposes of monthly reporting. The statistical summaries of reported deaths from all registries (communities) are pooled together into district level statistical summaries.

\section{Legal provisions on births and deaths registration}

Our review of legal and policy documents revealed that the Births and Deaths Registry Act, 1965, Act 301, stipulated that a central office be located in Accra (in this Act is referred to as "the central office") and a local office in such registration and local district (the central and local offices) as may be prescribed. We reproduce here, excerpts from the Act.

"The central office shall be the office of registration of all births and deaths in Ghana. The functions of the local offices shall be such as may be prescribed for purposes of recording of vital events. The central office and the local offices are collectively known as the Births and Deaths Registry. The Act provides that under the management and control of an officer called the Registrar of Births and Deaths, appointed by the Government, there shall be 
held in the Central Office, registers of all births, foetal deaths and deaths occurring in Ghana. The Act makes provision for the possibility of dividing the country into registration districts, and for the appointment of District Registrars and Assistant District Registrars (Figure 1).

\section{Recording, transfer and storage of vital events}

The Registration of Births and Deaths Act, 1965, Act 301 classifies death into two (2) distinct categories, namely foetal and adult deaths. This is based upon life-course from conception to time of death. For purposes of place of registration, the Act makes distinction between deaths on "High Seas/Air Space above High Seas" and deaths occurring on land defined as "Land Deaths". The Act specifies clearly, different procedural requirements in the instance of each category of deaths, either on land or on High Seas/Air Space above High Seas. Under Part Six (VI) tilted "miscellaneous" of the Act, the document recognizes "Fraudulent Registrations and Certificates", which when occur may have important implications on the overall quality of the information contained in the death registers. A brief description of the different categories of deaths as recognized by Act 301 and the specific requirements for their registration under laws of Ghana is presented as follows.

\section{Registration of foetal death}

For the purposes of the Act 301, a foetal death is death prior to the complete expulsion or extraction from its mother of a product of conception, irrespective of the duration of pregnancy. The death is indicated by the fact that after such separation the foetus does not breathe or show any other evidence of life, such as beating of the heart, pulsation of the umbilical cord, or definite movement of voluntary muscles. Every foetal death, which occurs in any district to which this Act applies, shall be registered as provided in it. Where foetal death occurs, the person who would have been responsible for the registration of a live birth shall furnish the prescribed statement regarding the foetal death. The person furnishing the required statement regarding the foetal death shall either: i) deliver to the Registrar a written certificate in respect of the foetal death signed by a registered medical practitioner or a midwife who was in attendance at the occurrence or who had examined the foetus; ii) make a declaration to the effect that no registered medical practitioner or midwife was present at the occurrence or had examined the foetus or that his or her certificate could not be obtained in respect of the foetal death.

If the Registrar is satisfied with the certificate or the declaration of cause of death, he shall register the foetal death and then issue a

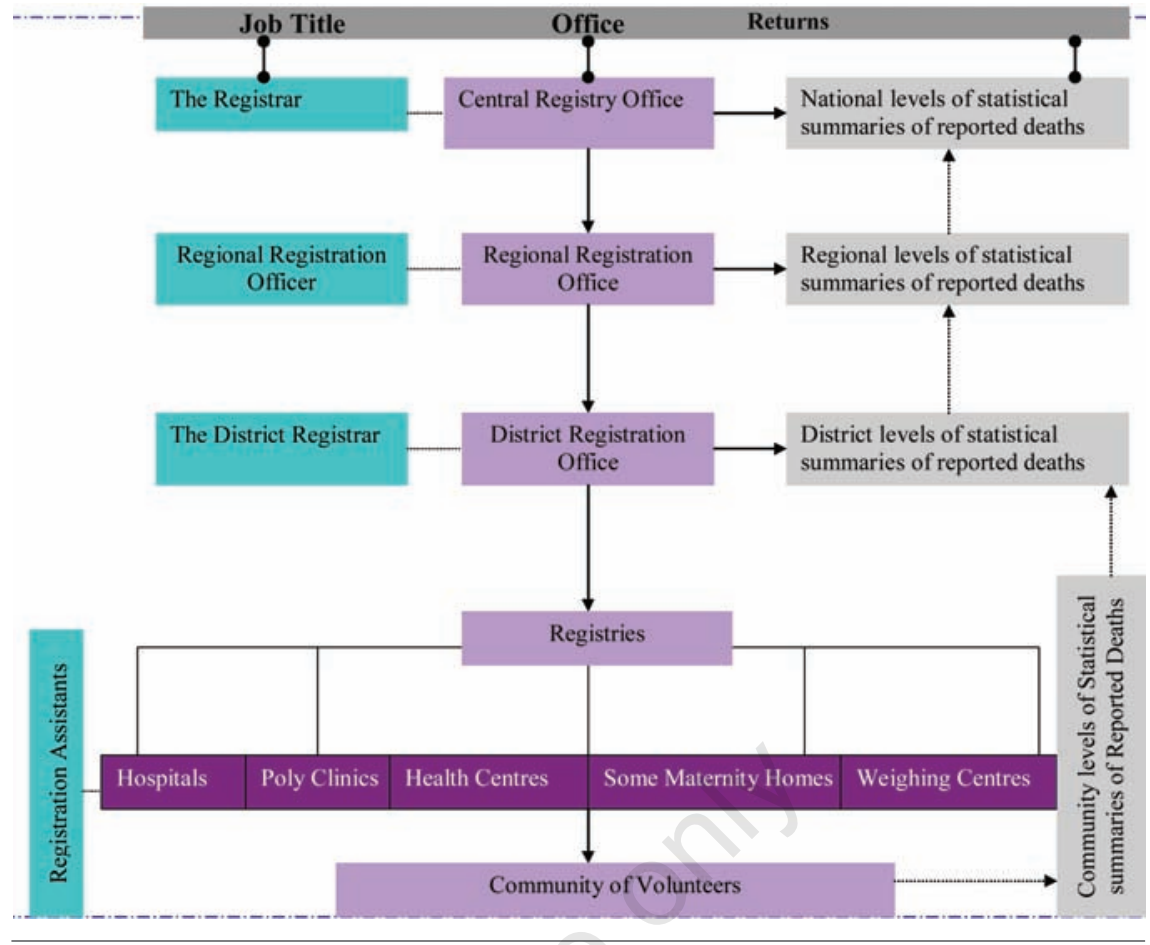

Figure 1. The operational set-up of the births and deaths registry in Ghana.

burial permit to the person requiring it for the purposes of burial or other deposition of the body.

\section{Registration of adult death}

Every death, which occurs in a district to which Act 301 applies, shall be registered by the Registrar of the District within twenty-four hours after the death and registration outside of the period shall only be made on payment of the prescribed fee. No death shall be registered after the expiration of twelve months from the date of death except with written authority of the Registrar of Births and Deaths and upon payment of the prescribed fee and note of such authority having been given shall be entered in the register.

Where a dead body is found and no information as to the place of death is available, the death shall be registered by the Registrar for the District in which the body is found.

It shall be the duty of each of the following persons to furnish the Registrar with the prescribed particulars of a death: i) the nearest relative of the deceased present at death or in attendance at the last illness of the deceased; ii) in default of the above, any relative of the deceased residing or being within the registration district; iii) if no relative is available, the occupier of the premises in which the death occurred; iv) any other person present at the death or having knowledge of the death; $v$ ) the coroner who has been notified of the death and has made inquiry or held an inquiry regarding the death.
The medical certificate stating the cause of death shall be issued free of charge by the medical practitioner who was last in attendance during the illness of the deceased, and the certificate shall be delivered to the Registrar.

When an inquiry is held on any dead body, the coroner who holds the inquiry shall complete and sign a certificate stating the cause of death and the certificate shall be delivered to the Registrar.

Where a death is a result of any circumstance in which a coroner is required under the coroners Act, 1960 (Act 18) to hold an inquiry or conduct a post-mortem examination, the Registrar shall neither register the death nor issue a death certificate unless ordered to do so by the coroner. Upon the receipt of the prescribed particulars, the Registrar, if satisfied as to the particulars or the certificate, as the case may be, shall register the death. The Registrar shall as soon as a death is registered issue: i) a burial permit free of charge in the prescribed form, and ii) a death certificate in the prescribed form on the payment of the prescribed fee.

The law requires that it shall not be lawful to bury a dead body elsewhere than in a burial ground authorized under Act 301, except with the permission of the Local authority. In order to provide the basis for this requirement, every local authority is mandated to provide public burial grounds for the burial of persons dying within the area of the local authority and to comply with any directions given by the 
Minister for this purpose.

The death records are then transported physically as forms, statistical summaries and registers from the registries in the communities to the Central Office, first through the district offices where they are forwarded to the Regional Offices. From the Regional Offices, they are finally forwarded to the Central Office in Accra as monthly returns (Figure 1) and from which national level statistical summaries of deaths are produced by cumulative summation of the regional level statistical summaries of mortalities.

Although the Act 301 establishing the Ghana Births and Deaths Registry provides complete inclusion of all deaths, the provision of death registration presents major challenges to data quality and does not guarantee that data are void of misclassification of cause of death and misallocation of place of death. This may present difficulty in establishing the burden of cause-specific mortality and give misleading results in spatial mortality analysis. Firstly, the Act does not indicate the technical competence and skill level of the Registrar who inspects the prescribed particulars and documents on dead bodies and in whose authority lies the granting of burial permits for both foetal and adult deaths. Secondly, the Act does not say anything about cause of death ascertainment process and gives no indication of how falsification of medical cause of death certificates may be checked and detected, although it recognizes "Fraudulent Registrations and Certificates". In rural areas where there are no controlled cemeteries, the lack of check could lead to many deaths escaping registration. Finally, some foetal deaths could go for burial without a statement of cause of death because the legal provisions under foetal statement such as "certificate cannot be obtained in respect of a given death". If this statement satisfies the Registrar, it means some individuals may conveniently provide justification for inability to obtain medical cause of death certificate.

\section{Transfer/transport of recorded events}

Transfer of death records from one level to another (say from the registries to the districts or from the district offices to the regional offices) occurs in two phases and the records are transported by motor vehicle in the form of three physical materials: i) log book or register which contains all information on the death events e.g. name of the deceased, nationality, normal place of residence, place of death, cause of death, etc.; ii) death registration forms where all the information in (1) above was recorded; iii) hardcopy of statistical summaries.

The statistical summaries and completed death registration forms are transported from one level to another on monthly basis, but the registers which are in the form of log books are transferred after they are full (this usually takes a year or so to be used up).

\section{Storage of recorded events}

The death records are held in hard files and as registers and stored in the archives of the Central Office. The completed registration forms are forwarded to the Government Statistician at the Ghana Statistical Services for subsequent analysis for policy planning."

\section{Results and Discussion}

Registration of births, recording deaths by age, sex and cause, calculating mortality levels and differentials and estimating population change are fundamental to evidence-based health policy, monitoring and evaluation of social interventions. ${ }^{2,20,22-25}$ It is widely believed that medically certified information is available for less than $30 \%$ of the estimated 50.5 million deaths that occur each year worldwide $^{2,20}$ and yet only very little effort has been made by national governments; especially those in developing countries such as Ghana, to strengthen these systems. Most experts agree that community population registration or SVR and DSS, when applied in conjunction with validated verbal autopsy procedures and implemented in a nationally representative sample of population clusters, represents an affordable, cost-effective, and sustainable short- and medium-term solution to this problem. ${ }^{2,20,23,25-27}$ In other words, SVR and DSS can complement other information sources by producing age-, sex-, and cause-specific mortality data that are continuous and more complete than those currently available. ${ }^{2,20}$ In reality, however, the tools and methods employed in an SVR system, are imperfect and require rigorous validation and continuous quality assurance. ${ }^{28}$ This makes sampling strategies for SVR a continuously evolving process. ${ }^{2}$

An assessment of the VRS in Ghana showed several structural and policy defects with important implications on the output data from the system. For example, Act 301 which gives authority to the Registrar for granting burial permits still had several deficiencies and did not guarantee elimination of misallocation of place of death. This imposed a major challenge to the use of the output data for spatial analysis of mortality. Misallocation of place of normal residence prior to death would effect the overall distribution of deaths and present a false impression of the risks of mortality.

Although the Ghana VRS is currently well structured, a major weakness remains that several communities lack registries and registration facilities, which means that the death registration would be incomplete, especially in rural areas with low coverage of mortality reporting. Another important weakness is that the registries are currently staffed with lowlevel staff without the specialized technical skills required to understand and appreciate the protocols and standard operating procedures as well as the intended scientific purpose of the output data from the VRS. The lack of robust designs for data collection, efficient methods for capturing data, standard data-collection instruments, coding specifications, query guidelines and standardized definitions in the Ghana VRS would raise legitimate questions about the quality of its output data.

Our analyses revealed severe deficiencies in the manner in which data captured by the registration system are stored. The traditional paper-based practices used in mortality reporting present major constraints to the generation of high quality data from the VRS in Ghana. For instance, we observed that the paper on which death records were stored was non-durable material with a short life-cycle. Information recorded on paper cards could easily be destroyed upon decay of the paper, especially through handling by multiple users and lifting from one point to another. The VRS is presently not automated and had no networks and linkages among the different registration points. This renders data transmission time from primary collection points to the central office inordinately prolonged providing avenues for data losses.

Additionally, the fact that the legal provisions guiding the conduct of checks on ascertainment of cause of death for the Ghana registration system did not indicate how the accuracy of the information on medical cause of death certificates would be verified, could lead to misclassification of cause of death in some instances. The probability of misclassification occurring in this way would particularly be high in rural areas where there are no controlled cemeteries and burials were unregulated. The probability of this occurring in urban areas would be very low and limited to urban periphery because of strict controls on burials in the core urban areas. With 10 years of experience in the implementation of DSS in Ghana, expertise required to implement low-cost reengineering work on the Ghana VRS that would improve the quality of output data is now widely available. Although it was noted that some attempts had been made to strengthen the Ghana VRS so as to improve the quality of its output data by way of introduction of the concept of "community population registers" and digitization of data at the central office, this would be counterproductive if the process is not supported by upgrade of workforce skills in the registration system and attraction of specialized skills. The workforce in the Ghana VRS at this time lack specialized skills required to implement highly technical protocols and standard procedures that would gen- 
erate high quality health data. A way to resolve the low skill problems in the Ghana VRS would be to recruit highly qualified professionals, (e.g. demographers, epidemiologists, sociologists, clinicians and economists, just as the case for DSS) into the sector.

Nonetheless, such a strategy would impose additional financial burden as salaries would have to be raised to accommodate and retain the specialized skilled personnel. However, this additional financial burden could be avoided if registration districts were developed into satellite field research centers which could then be absorbed by universities, research institutions, etc for use as "community population laboratories" for field research. Finally, it could also be possible that socioeconomic and religious factors might be associated with death registration in some communities and this could raise legitimate questions about registration completeness. For instance, in largely Muslim communities in Ghana, normal practice after death occurs is immediate burial. Therefore, if death registration processes required longer period than could be accommodated by this religious demand, then some deaths could escape cause of death ascertainment and their registration altogether However, this would not select in favor of one specific-cause over another and would not result in differential cause-specific reporting. Also, in low-income communities where residents might be too poor to worry about registration of deaths in lieu of struggling for survival, this situation could be characterized by illegal burials without registration. When this happens, it might present some selection bias against poverty related cause-specific mortalities. This might mean that socioeconomic inequalities could affect death registration in a sense that poverty related cause-specific mortalities would escape registration as it were.

\section{Overall usefulness of routine data from the Ghana vital registration systems}

Though death registration coverage was observed to be low, data could still be used because such limited provision did not select in favor of any particular cause of death over the other despite the fact that some marginal systematic biases might be occurring. The assumption is premised on the fact that cause of death is constant all-year round, except during epidemics, disasters, wars, etc and that it is fact of death and not cause of death matters in death registration. This assumption could be exploited to surmount the systematic biases associated with low coverage in death registration, misclassification and misallocation of cause of death. This could be achieved through the application of various epidemiologic procedures including the use of standardized mor- tality ratio (SMR) and proportional mortality ratio (PMR) techniques in the analysis of the limited data. Furthermore, the systematic bias occurring as a consequence of differing socioeconomic levels could be accommodated if communities were grouped into income strata for independent analyses. Finally, although illegal burials might still occur within the Ghana VRS, such practices are not intended to skip reporting any cause of death over the other, but to avoid cumbersome procedures required to obtain burial certificates and could be accounted for by standard analytic methods. The problem of incomplete registration would normally be addressed through the application of direct and indirect standardization procedures.

\section{Conclusions}

A careful assessment of the policy documents and operational procedures of the Ghana VRS showed the presence of some lapses in the system that could undermine the validity of the output data. Considering the outdated registration practices (paper-based registration) used in Ghana's VRS, a significant amount of the data captured and stored could be lost. Errors associated with such data losses would normally class under random or non-differential biases. Additionally, it might be possible that the current setup of the Ghana VRS, the low skill level of its workforce and the law governing its operations could allow for misclassification of cause of death and misallocation of place of death. The authors are convinced that restructuring and strengthening of institutional structures, upgrade of workforce skills (i.e. outsourcing the services of highly qualified professional such as demographers, epidemiologists, sociologists, clinicians and economists) and policy reforms in the Ghana VRS could strengthen the institutional structure and thereby result in significant improvement in data collection functionalities. This should lead to significant improvement in the quality of the output data.

Our general conclusion is that the main problems associated with VRS dysfunction are not whether to stick to the paper-based system which of course is not efficient, or to switch to an electronic system totally, but rather, developing a system that will use the two systems complementarily in a coherent and coordinated manner as reported in other studies elsewhere.

Finally, the specific conclusion is that the lapses in the registration system could potentially introduce some systematic biases. However, the nature of the biases associated with the registration system are such that the data may still be used in health analysis with- out much worry about validity problems through careful application of standard epidemiologic techniques.

\section{Recommendations}

It is the view of authors that if the Ghana Statistical Service (GSS), in collaboration with the Ghana Health Service (GHS), implemented the following strategies would greatly strengthen the Ghana VRS and therefore lead to complete elimination or substantial reduction in the data quality problems associated with the system. For instance, the status of the Births and Deaths Registry could be elevated to a Department status (Department or Bureau of Routine Statistics - DRS) and staff salaries adjusted accordingly upwards to attract highly skilled and specialized work force (e.g. demographers, epidemiologists, biostatisticians, etc) with competencies required to handle very complex data collection designs and highly sophisticated data collection protocols. To improve efficiency in data generation, reproducibility, reduce transmission uncertainties and storage difficulties as well as minimize data losses, then the new DRS could consider implementing an electronic automation or computerize and network the registration system which connects all the registration centers/points across the entire country. Such a computerized system would increase registration coverage without necessarily increasing staff strength and at the same time minimize the chance of data losses through outdated paper based transportation from one point to another. Additionally, an amendment to the Act establishing the VRS could be considered so as to regulate falsification of cause of death certificates, improve strategies for accurate recording of deceased place of address, and the introduction of stricter controls of cemeteries and a more effective cause of death ascertainment verification strategies at the Coroner Court. This would significantly reduce the frequency of misclassification of cause of death and misallocation of place of death.

\section{References}

1. Morrow RH Jr. The application of a quantitative approach to the assessment of the relative importance of vector and soil transmitted diseases in Ghana. Soc Sci Med 1984;19:1039-49.

2. Setel PW, Sankoh 0, Rao C, et al. Sample registration of vital events with verbal autopsy: a renewed commitment to measuring and monitoring vital statistics. Bull World Health Organ 2005;83:611-7. 
3. Bahl R, Frost C, Kirkwood BR, et al., Infant feeding patterns and risks of death and hospitalization in the first half of infancy: multicentre cohort study. Bull World Health Organ 2005;83:418-26.

4. Mackenbach JP, Bos V, Garssen MJ, Kunst AE. Mortality among non-western migrants in The Netherlands. Ned Tijdschr Geneeskd 2005;149:917-23.

5. Afukaar FK, Antwi P, Ofosu-Amaah S. Pattern of road traffic injuries in Ghana: implications for control. Inj Control Saf Promot 2003;10:69-76.

6. London J, Mock C, Abantanga F.A, et al. Using mortuary statistics in the development of an injury surveillance system in Ghana. Bull World Health Organ 2002; 80:357-64.

7. Boulle A, Chandramohan D, Weller P. A case study of using artificial neural networks for classifying cause of death from verbal autopsy. Int $\mathrm{J}$ Epidemiol 2001;30: 515-20.

8. Chandramohan D, Setel P, Quigley M. Effect of misclassification of causes of death in verbal autopsy: can it be adjusted? Int J Epidemiol 2001;30:509-14.

9. Afzal, M. Some considerations for demographic assessment of developing countries. Pak Dev Rev, 1986;25:517-30.

10. Chasteland JC. Problems of demographic data collecting in Arab countries of the Middle East. Egypt Popul Fam Plann Rev 1970;3:29-40.

11. Dougan S, Harris JP. Overcounting of black Africans in the UK: the problem of undetected record duplication. Commun Dis Public Health 2003;6:147-51.
12. Songsore J, Goldstein G. Health and Environment Analysis for DecisionMaking (HEADLAMP): field study in Accra, Ghana. World Health Stat Q 1995;48:10817.

13. Petit PL, van Ginneken JK. Analysis of hospital records in four African countries, 1975-1990, with emphasis on infectious diseases. J Trop Med Hyg 1995;98:217-27.

14. Tettey Y, Wiredu EK. Autopsy studies on still births in Korle Bu Teaching Hospital: pathological findings in still births and their placentae. West Afr J Med 1997;16: 12-9.

15. Timæus IM. Impact of HIV/AIDS on mortality in sub-Sahara Africa evidence from national surveys and censuses. AIDS 1998;12:S15-27.

16. Timaeus I, Graham W. Measuring Adult Mortality in Developing Countries: A Review and Assessment of Methods. The World Bank, Policy Research Working Paper Series, no. 155, 1998.

17. Timaeus IM. Adult Mortality: Levels, Trends, and Data Sources. In: Disease and Mortality in Sub-Sahara Africa. DT Jamison, RG Feachem, MW Makgoba, ER Bos, FK Baingana, KJ Hogman, KO Rogo (eds). 1991, The World Bank Publ., Washington, DC, USA.

18. Wiredu EK, Tettey Y. Autopsy studies on still births in Korle Bu Teaching Hospital. II: Causes of death in 93 still births. West Afr J Med 1998;17:148-52.

19. Baiden F, Hogdson A, Binka FN. Demographic surveillance sites and emerging challenges in international health. Bull World Health Organ
2006;84:163.

20. Chandramohan D, Shibuya K, Setel P, et al. Should Data from Demographic Surveillance Systems Be Made More Widely Available to Researchers? PLoS Med 2008;5:e57.

21. Lucas D, Meyer P. Beginning of Australian population studies. 2003, The Australian National University, Canberra, Australia.

22. Allotey PA, Reidpath D. Information quality in a remote rural maternity unit in Ghana. Health Policy Plan 2000;15:170-6.

23. de Beer M, Padayachee GN, Ijsselmuiden C, Coetzee D. The reliability of mortality data in Johannesburg. S Afr Med J 1993;83:597-601.

24. Hessel PA, Hnizdo E, Goldstein B, SluisCremer GK. Pathological findings in mine workers: II. Quality of the PATHAUT data. Am J Ind Med 1987;12:81-9.

25. Lerer LB, Improving mortality data in South Africa: review of next of kin statements to determine cause of death in police certification. J Epidemiol Community Health 1993;47:248-50.

26. Hyder AA, Rotllant G, Morrow RH. Measuring the burden of disease: healthy life-years. Am J Public Health 1998;88:196202.

27. Wiley AS, Pike Il. An alternative method for assessing early mortality in contemporary populations. Am J Phys Anthropol 1998; 107:315-30.

28. Krickeberg K. Principles of health information systems in developing countries. HIM J 2007;36:8-20. 